\title{
ITPA:C.94C>A and NUDTI5:C.4I5C>T Polymorphisms and Their Relation to Mercaptopurine-Related Myelotoxicity in Childhood Leukemia in Thailand
}

\author{
Boonchai Boonyawat (iD) \\ Chalinee Monsereenusorn $\left(\mathbb{D}^{2}\right.$ \\ Apichat Photia (D) ${ }^{2}$ \\ Nawachai Lertvivatpong ${ }^{2}$ \\ Varissara Kaewchaivijit ${ }^{3}$ \\ Punyanuch Jindatanmanusan ${ }^{2}$ \\ Piya Rujkijyanont $\mathbb{( D}^{2}$ \\ 'Division of Medical Genetics, \\ Department of Pediatrics, \\ Phramongkutklao College of Medicine \\ and Phramongkutklao Hospital, Bangkok, \\ Thailand; ${ }^{2}$ Division of Hematology- \\ Oncology, Department of Pediatrics, \\ Phramongkutklao College of Medicine \\ and Phramongkutklao Hospital, Bangkok, \\ Thailand; ${ }^{3}$ Department of Pediatrics, \\ Phramongkutklao College of Medicine \\ and Phramongkutklao Hospital, Bangkok, \\ Thailand
}

Correspondence: Piya Rujkijyanont Department of Pediatrics, Phramongkutklao College of Medicine and Phramongkutklao Hospital, 315

Ratchawithi Road, Ratchathewi, Bangkok, 10400, Thailand

Tel/Fax +66 2644 4130

Email piya_rujk@yahoo.com
Background: Mercaptopurine is a key agent in childhood leukemia treatment. Genetic polymorphism in the genes involving thiopurine metabolisms is related to 6-MP related toxicity.

Objective: This study aimed to determine the prevalence of ITPA:c.94C $>A$ and NUDT15: c. $415 C>T$ polymorphisms among Thai children diagnosed with leukemia and their association with mercaptopurine-related myelotoxicity.

Methods: Patients and survivors with a diagnosis of leukemia treated with mercaptopurinecontaining chemotherapy regimens were enrolled. Clinical data and laboratory parameters during treatment as well as ITPA:c. $94 C>A$ and NUDT15:c.415C $>T$ genotypes were analyzed.

Results: In all, 99 patients with acute leukemia or survivors were enrolled in the study. The prevalences of ITPA:c.94C $>A, N U D T 15: c .415 C>T$, and co-occurrence of ITPA:c.94C $>A$ and NUDT15:c. 415C $>T$ polymorphisms were 34,17 , and 4\%, respectively. Numbers of absolute neutrophil count (ANC) and platelet count significantly decreased among patients carrying NUDT15:c. $415 C>T$ compared with NUDT15 wild type patients with $p$-values $<0.001$ and 0.019 , respectively. The differences were not observed among patients carrying ITPA: c.94C $>A$ compared with ITPA wild type patients. According to multivariate GEE, NUDT15:c. 415C $>T$ and co-occurrence of ITPA:c.94C $>A$ and NUDT15:c. $415 C>T$ had a significant negative effect on ANC during treatment (coefficient: -463.81 ; CI: -778.53 , -149.09 ; $p$-value $=0.004$ and coefficient: -527.56 ; CI: $-1045.65,-9.48 ; p$-value $=0.046$ ). No significant effect of ITPA:c.94C $>A$ on ANC during treatment was observed.

Conclusion: ITPA:c.94C $>A$ and NUDT15:c.415C $>T$ polymorphisms are common among Thai children with leukemia. A strong association with mercaptopurine-related myelotoxicity was observed among patients carrying either NUDT15:c.415C $>T$ alone or combined with ITPA:c. $94 C>A$.

Keywords: NUDT15, ITPA, 6-MP, myelotoxicity, pediatric oncology

\section{Background}

The survival rate of childhood cancer has dramatically improved over the past decades. ${ }^{1}$ The key elements attributing to this successful achievement include early recognition and initial management of cancer as well as its complications, newly developed cancer treatment strategies and, most importantly, improved supportive care throughout the length of cancer treatment. Pediatric hematologic malignancies, 
including leukemia and lymphoma, are considered as prototypes of curative cancer. ${ }^{2-5}$ Despite the favorable outcomes, significant numbers of patients still encounter treatment-related toxicities. Systemic chemotherapy incorporated in most pediatric cancer therapeutic regimens contains cytotoxic effects involving not only cancerous cells, but also normal growing cells resulting in suppression of hematopoiesis and eventually leading to anemia, hemorrhage, and a higher risk of serious infection from decreased red blood cell, platelet, and white blood cell production, respectively. ${ }^{6,7}$

Mercaptopurine (also referred as 6-mercaptopurine or 6$\mathrm{MP}$ ) is a prodrug of a purine analog functioning as an antagonist to endogenous purines required for DNA synthesis and replication during the S-phase of the eukaryotic cell cycle as well as inhibiting RNA and protein synthesis. ${ }^{89}$ The drug has been implemented as a key agent in childhood acute lymphoblastic leukemia (ALL) treatment regimens and also been used in other therapeutic regimens for childhood lymphoblastic lymphoma and acute promyelocytic leukemia (APL). Related studies reported that the tolerance to 6-MP varies among each individual patient, in which the most common adverse effect of the drug is myelosuppression with the onset at 7-10 days, nadir at 14 days, and recovery occurring by 21 days. ${ }^{10,11}$ Pathogenesis of this discrepancy of the drug-related toxicity seems to be related to genetic polymorphisms in the genes involved in thiopurine metabolisms. ${ }^{12}$

Genetic polymorphisms in the gene encoding thiopurine methyltransferase (TPMT) are widely accepted to be involved in thiopurine metabolisms resulting in 6-MP related myelotoxicity; however, geographic variation of frequencies and distributions of variant TPMT alleles have been reported in which the allelic frequencies were much lower in Asian compared with Caucasian populations. ${ }^{13-15}$ On the other hand, genetic polymorphisms in other genes encoding enzymes involved in thiopurine metabolisms including inosine triphosphate pyrophosphohydrolase (ITPA) and nucleoside diphosphate linked moiety X-type motif 15 (NUDT15) has been reported to be more evident in Asian compared with Caucasian populations. ${ }^{16-18}$ ITPase is an enzyme involved in the hydrolysis of thioinosine triphosphate (TITP) to thioinosine monophosphate (TIMP) in which the deficiency of this enzyme among patients receiving 6-MP could result in toxic accumulation of TITP. ${ }^{16,19}$ However, the clinical correlation between ITPA genotypes and 6-MP related toxicity remains controversial and is not routinely applied to clinical practice. The NUDT15 protein is an enzyme involved in thiopurine metabolisms by catalyzing the conversion of toxic thioguanine triphosphate to less toxic thioguanine monophosphate, resulting in a reduced cytotoxic effect of 6-MP. ${ }^{16,19}$ Therefore, genetic polymorphisms of ITPA and NUDT15 could potentially lead to 6-MP related cytotoxicity effects.

Herein, we conducted a prospective cross-sectional observational study to determine the prevalence of NUDT15 and ITPA polymorphisms among Thai children diagnosed with acute leukemia and their association with 6-MP related myelotoxicity.

\section{Methods}

\section{Patient Selection}

Pediatric oncology patients undergoing chemotherapy at the Division of Hematology and Oncology, Department of Pediatrics, Phramongkutklao Hospital from January 1, 2018 to January 31, 2020 were enrolled in this study. Written informed consent and assent forms to participate in the study were obtained from all participants including the children themselves as well as their parents or legal guardians before engaging in the study. This prospective study was approved by the Institutional Review Board, Royal Thai Army Medical Department according to the ethics principles of the Declaration of Helsinki (1975) and its revision (reference number: IRBRTA 799/2562). The study was also registered and approved by the Thai Clinical Trials Registry (TCTR20190828003). The study's inclusion criteria included patients aged less than 18 years receiving a diagnosis of acute leukemia and undergoing 6-MP containing chemotherapeutic regimens or pediatric leukemia survivors who had a history of receiving the treatment regimens. The study's exclusion criteria consisted of patients with a history of allergy or contra-indication to 6-MP, unanalyzable medical information due to poor documented medical records, or those who denied having blood samples for ITPA and NUDT15 polymorphisms obtained.

\section{Outcome Measurement}

The study adhered to the Preferred Reporting items for Observational studies in Endodontics (PROBE) guidelines. The study schema is shown in the flow diagram in Figure 1. One hundred and three patients were initially recruited to the study; however, four were excluded and 99 consented and enrolled in the study. The primary outcome of this study was to describe the prevalence of ITPA and NUDT15 polymorphisms among pediatric leukemia patients treated with 6-MP containing chemotherapeutic regimens. The secondary outcomes were to determine the 


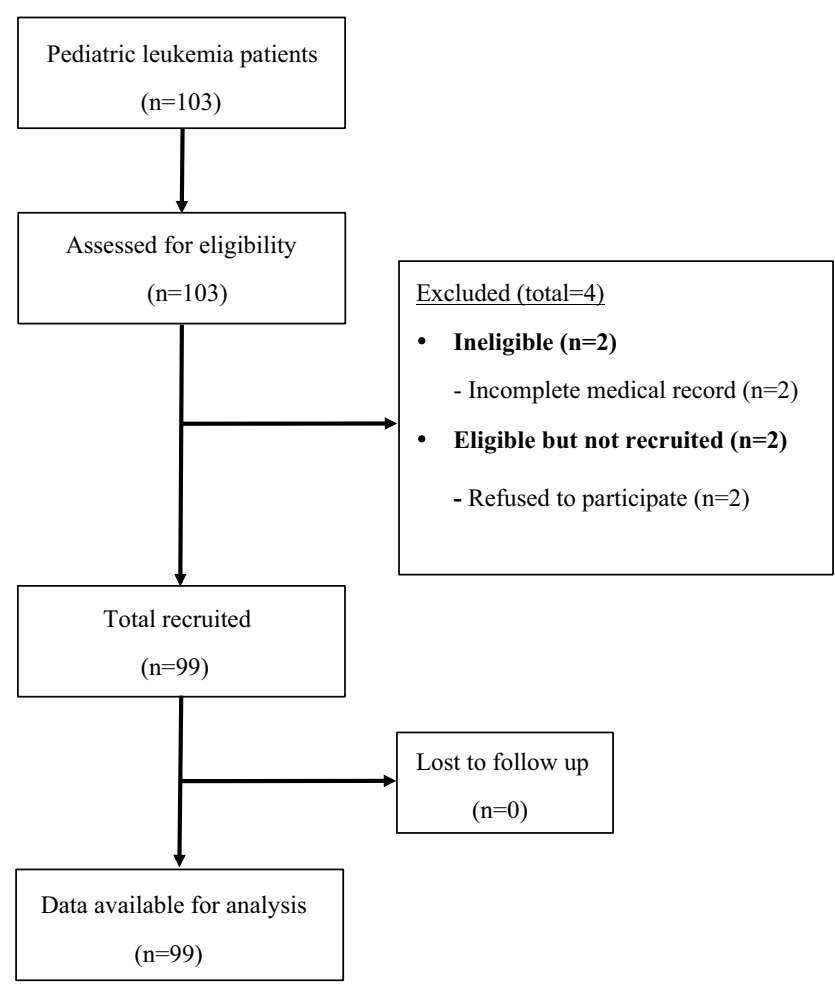

Figure I Study flow diagram.

association between ITPA and NUDT15 polymorphisms and 6-MP related myelotoxicity. The study defined neutropenia as an absolute neutrophil count (ANC) of less than 1,500 cells $/ \mathrm{mm}^{3}$ and myelotoxicity as an adverse effect of cancer treatment in which bone marrow activity is decreased resulting in decreased numbers of red blood cells, white blood cells, and platelet count.

\section{6-MP Containing Chemotherapy Regimens}

According to the national protocols for childhood cancers conducted by the Thai Pediatric Oncology Group (ThaiPOG), 6-MP was administered at the dose of $50 \mathrm{mg} /$ $\mathrm{m}^{2} /$ dose orally once daily during the maintenance phase of acute lymphoblastic leukemia protocols for a total duration of 20 months among females and 32 months among males. The 6-MP dose of $50 \mathrm{mg} / \mathrm{m}^{2} /$ dose was also administered to patients receiving a diagnosis of APL during the maintenance phase of APL protocol for a total duration of 24 months. All patients were followed up at the Oncology Clinic and had complete blood count (CBC) measured monthly throughout the duration of treatment. 6-MP dosing would then be adjusted on each individual patient based on the results of ANC and platelet count.

\section{Identification of ITPA:c.94C $>A$ and NUDTI 5:c.4I5C>T Polymorphisms}

After informed consent was obtained from the patients and their parents, genomic DNA was extracted from peripheral blood leukocytes using commercial kits following manufacturer instructions. The ITPA:c. $94 C>A$ and NUDT15: c. $415 C>T$ fragments were amplified by PCR using the primers as previously described. ${ }^{20,21}$ Each $50 \mu \mathrm{L}$ PCR mixture contained $1.5 \mathrm{mM} \mathrm{MgCl} 2,200 \mu \mathrm{M}$ of each dNTP, $0.2 \mu \mathrm{M}$ of each primer, 100-200 ng of genomic DNA and 1 units Taq DNA polymerase. PCR amplification started with initial denaturation at $95^{\circ} \mathrm{C}$ for 5 minutes, and continued with 30 cycles of $95^{\circ} \mathrm{C}$ for 20 seconds, $55^{\circ}$ $\mathrm{C}$ for 20 seconds, and $72^{\circ} \mathrm{C}$ for 30 seconds. Final extension was performed at $72^{\circ} \mathrm{C}$ for 5 minutes.

A 328-bp PCR product for ITPA:c.94C $>$ A and 269-bp product for NUDT15:c.415C $>$ T were digested with $N s p \mathrm{I}$ and TaaI restriction enzymes, respectively. The ITPA: c. $94 \mathrm{C}>\mathrm{A}$ allele yielded two fragments of $238 \mathrm{bp}$ and 90 bp; whereas, the NUDT15:c.415C $>\mathrm{T}$ allele yielded $142 \mathrm{bp}$ and $127 \mathrm{bp}$ after digestion. The PCR products were resolved in $2 \%$ agarose gels, as shown in Figure 2. Sanger sequencing was also performed on several random samples which were positive and negative for restriction enzyme digestion, and the results were $100 \%$ concordance.

\section{Statistical Analysis}

Baseline values of selected variables were analyzed and presented as mean with standard deviation (SD) or median (range) for continuous variables and calculated using frequency and percentage for categorical variables. Comparisons between two independent data sets were analyzed using Fisher's exact test for categorical data and independent sample $t$-test or Mann-Whitney $U$-test for continuous data. Longitudinal data from time-dependent variables were analyzed using a multivariate generalized estimating equation (GEE) to examine the associations between ITPA and NUDT15 polymorphisms as well as 6-MP administrative dosing and ANC measured monthly during the maintenance phase of acute leukemia protocols. With GEE, the relationships between the variables of the model at different time-points were analyzed simultaneously. STATA/MP, Version 12 Software (STATA Corp., TX, USA) was used and a $p$-value $<0.05$ was considered statistically significant. 

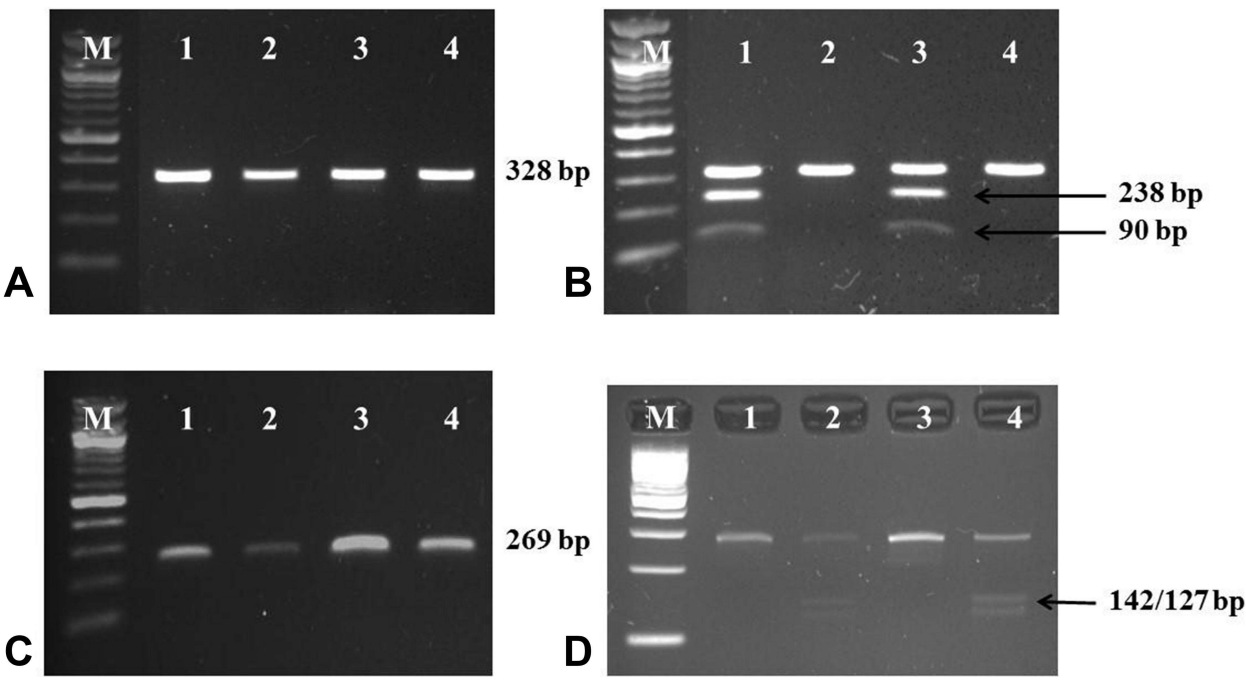

Figure 2 Gel electrophoresis of 328-bp PCR products of ITPA:c.94C>A variant (A) before and (B) after Nspl restriction enzyme digestion and 269-bp PCR products of NUDTI 5:c.4I5C>T variant (C) before and (D) after Taal restriction enzyme digestion, respectively (lane I-4, M-I00 bp marker). The 328-bp PCR products of ITPA:c.94C>A variant were digested by Nspl and yielded 238-bp and 90-bp fragments as shown in lane I and 3; (B) suggesting the heterozygous for ITPA:c.94C>A variant. The 269 bp-PCR products of NUDTI5:c.4I5C>T variant were digested by Taal and yielded I42-bp and I27-bp fragments as shown in lane 2 and 4; (D) indicating the heterozygous for NUDT I 5:c. 4I5C>T variant.

\section{Results}

\section{Patient Characteristics}

Patient characteristics including age, sex, leukemia type, central nervous system (CNS) status, ploidy from conventional cytogenetic analysis, and risk stratification for ALL and disease status at enrollment are summarized in Table 1. Most participating patients were younger children of preschool age. Males were more predominant than females at a ratio of 1.3:1. Patients with various subtypes of acute leukemia were enrolled in this study. The most common leukemia subtype was pre-B ALL followed by T-ALL resembling a typical leukemia distribution among pediatric patients. Both APL and mixed phenotype acute leukemia (MPAL) were less common and rare subtypes of pediatric acute leukemia, respectively, and only one patient with APL and one with MPAL were enrolled in this study. Most patients had a normal karyotype with no CNS involvement at initial diagnosis and successfully achieved complete remission at the time of enrollment to this study. In addition, most ALL patients were stratified as standard and high risks; however, the 6-MP dose during the maintenance phase of standard and high risk ALL protocols was similar to the dose in a very high-risk ALL protocol.

\section{Prevalence of ITPA:C.94C>A and NUDTI5:}

\section{4 I5C $>T$ Polymorphisms}

PCR-Restriction Fragment Length Polymorphism (PCRRFLP) performed on the genomic DNA obtained from 99 enrolled patients with acute leukemia was used to identify ITPA:c. $94 C>A$ and NUDT15:c.415C $>T$ polymorphisms and the results are summarized in Table 2. Interestingly, 47 patients (47\%) were found to be heterogeneous for either one of these two polymorphisms. The prevalences of ITPA:c. $94 C>A$ and NUDT15:c. 415C $>T$ polymorphisms among pediatric patients with leukemia in this study were $34 \%$ and $17 \%$, respectively, and $4 \%$ of the patients were found to have co-occurrence of ITPA:C. $94 C>A$ and NUDT15:c. $415 C>T$ polymorphisms.

\section{Patient Characteristics According to ITPA and NUDTI 5 Genotypes}

As shown in Table 3, various demographic characteristics including age, sex, leukemia type, CNS status, ploidy from conventional cytogenetic analysis, risk stratification for ALL and disease status at enrollment were described among participating patients with and without ITPA:c.94C $>A$ and NUDT15:c. $415 C>T$ polymorphisms. No differences were found regarding clinical information between patients carrying ITPA and NUDT15 wild types versus those carrying ITPA:c. $94 C>A$ and NUDT15:c. $415 C>T$ polymorphisms.

Association Between ITPA:c.94C $>A$ and NUDT I5:C.4I5C>T Polymorphisms and 6MP Related Myelotoxicity

The effects of ITPA:c. $94 C>A$ and NUDT15:c.415C $>T$ polymorphisms on bone marrow toxicity were determined 
Table I Patient Demographic Data

\begin{tabular}{|l|c|}
\hline Patients (n=99) & N (\%) \\
\hline Age at diagnosis (years) & $5.1 \pm 4.4$ \\
Mean \pm SD & $4.5(0.3-18.0)$ \\
Median (min-max) & \\
\hline Gender & $55(56)$ \\
Male & $44(44)$ \\
Female & \\
\hline Diagnosis & $87(88)$ \\
Pre-B ALL & $10(10)$ \\
T-ALL & $1(1)$ \\
MPAL & $1(1)$ \\
APL & \\
\hline CNS status & $91(92)$ \\
CNS-I & $6(6)$ \\
CNS-2 & $2(2)$ \\
CNS-3 & $83(84)$ \\
\hline Ploidy & $16(16)$ \\
Normal & $95(96)$ \\
Hyperdiploidy & $4(4)$ \\
\hline Risk stratification for ALL (n=97) & \\
Standard & $47(48)$ \\
High & $42(43)$ \\
Very high & $8(8)$ \\
\hline Disease status & \\
Remission & \\
Relapse & \\
\hline Not Data arepresentedasmeat & \\
\hline
\end{tabular}

Note: Data are presented as mean $\pm \mathrm{SD}$ and median (range) for continuous variables and number (\%) for categorical variables.

Abbreviations: ALL, acute lymphoblastic leukemia; ANC, absolute neutrophil count; APL, acute promyelocytic leukemia; CNS, central nervous system; MPAL, mixed phenotype acute leukemia.

Table 2 Prevalence of ITPA:c.94C>A and NUDTI5:C.4I5C>T Polymorphisms

\begin{tabular}{|l|c|}
\hline Genetic Polymorphisms & N (\%) \\
\hline ITPA:c.94C>A & $65(66)$ \\
Wild type & $34(34)$ \\
Heterozygous genotype & \\
\hline NUDTI5:c.4 I5C>T & $82(83)$ \\
Wild type & $17(17)$ \\
Heterozygous genotype & \\
\hline Both ITPA:c.94C>A and NUDTI5: & \\
c.4I5C>T heterozygous genotypes & $4(4)$ \\
Yes & $95(96)$ \\
No & \\
\hline
\end{tabular}

Note: Data are presented as number (\%) for categorical variables.

Abbreviations: ITPA, inosine triphosphate pyrophosphohydrolase; NUDTI5, nucleoside diphosphate linked moiety X-type motif 15. using the mean numbers of ANC and platelet counts as well as hemoglobin levels measured on each cycle of chemotherapy during the maintenance phase of leukemia treatment protocols, as shown in Figure 3. Interestingly, no differences were observed concerning ANC, platelet count, and hemoglobin levels between patients carrying ITPA:c. $94 C>A$ polymorphism and those carrying ITPA wild type (Figure $3 \mathrm{~A}-\mathrm{C}$ ). However, the numbers of ANC and platelet counts were significantly decreased among patients carrying NUDT15:c. $415 C>T$ polymorphism compared with those carrying NUDT15 wild type with $p$-values $<0.001$ and 0.019 , respectively (Figure $3 \mathrm{E}$ and F). In addition, 6-MP administrative dosing among patients carrying NUDT15:c.415C $>T$ polymorphism was also significantly less compared with the dose among wild type patients with a $p$-value $<0.001$ (Figure $3 \mathrm{H}$ ). In contrast, the significant decrease of daily 6-MP administrative dosing among patients carrying ITPA wild type ( $p$ value $=0.007$ ) could be from patients presenting the NUDT15:c.415C $>T$ being included in this group (Figure 3D).

Moreover, multivariate GEE was used to identify the relationship between ITPA:c.94C $>A$ and NUDT15: c. $415 C>T$ polymorphisms as well as 6-MP administrative dosing and ANC measured monthly during the maintenance phase of acute leukemia protocols as described in Table 4. Interestingly, ITPA:c. $94 C>A$ polymorphism and 6-MP administrative dosing among patients presenting this specific genetic polymorphism did not have a significant effect on monthly ANC during treatment with $p$-values of 0.948 and 0.062 , respectively (Table 4, Model 1). On the other hand, both NUDT15:c.415C $>T$ polymorphism and 6MP administrative dosing among patients presenting this specific genetic polymorphism were found to have a significantly negative effect on monthly ANC during treatment (coefficient: -463.81 ; CI: $-778.53,-149.09$ and coefficient: -9.29 ; CI: $-18.05,-0.52$ ) with $p$-values of 0.004 and 0.038 , respectively (Table 4, Model 2). Moreover, co-occurrence of ITPA:c. $94 C>A$ and NUDT15:c.415C>T polymorphisms was also found to have a significant negative impact on monthly ANC (coefficient: -527.56; CI: -1045.65, -9.48) with a $p$-value of 0.046 . However, no significant association was observed between 6MP administrative dosing among patients presenting co-occurrence ITPA: c. $94 C>A$ and NUDT15:c. $415 C>T$ polymorphisms and 


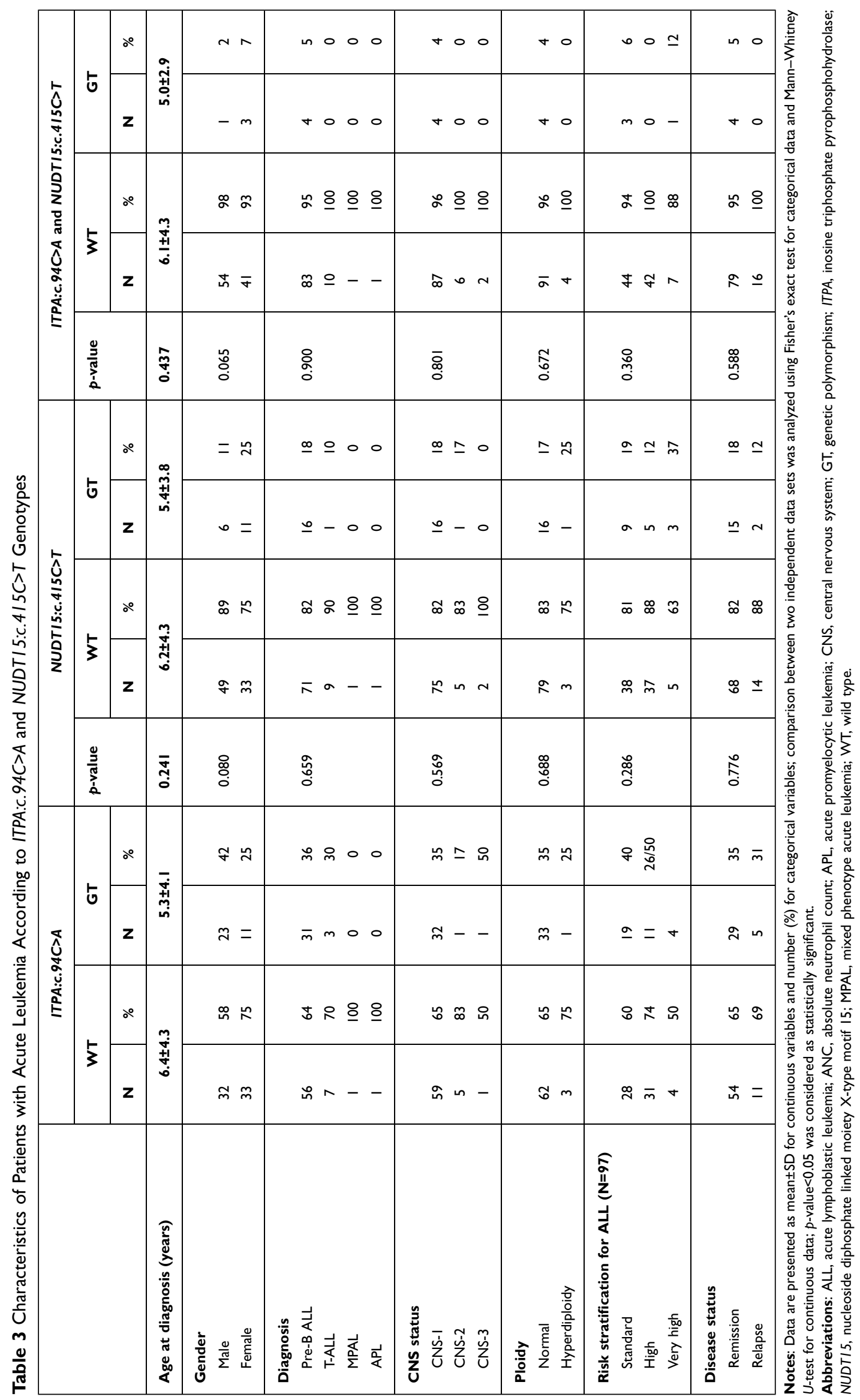


A

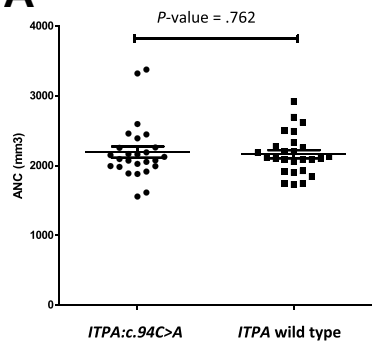

E

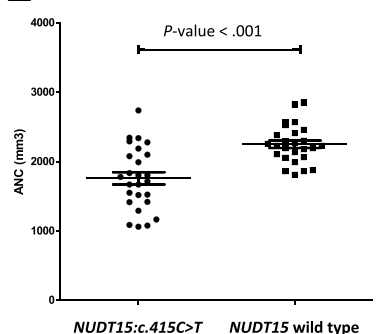

B

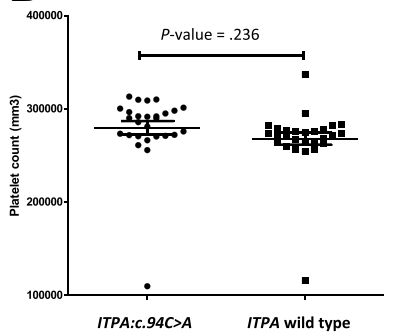

F

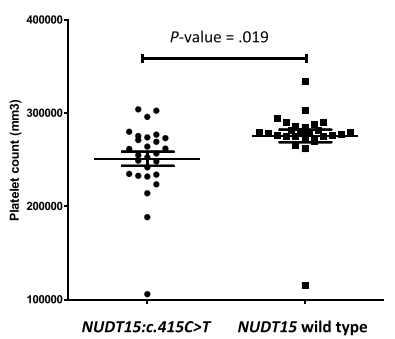

C

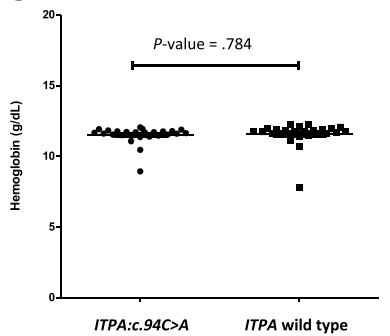

G

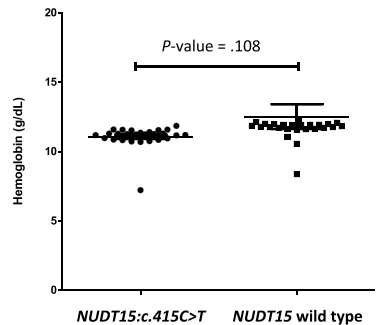

D

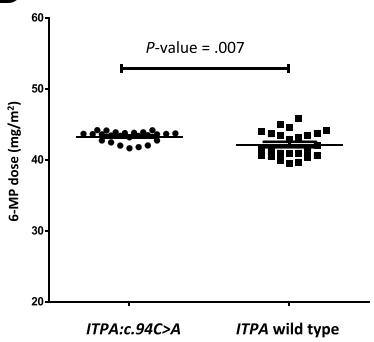

H

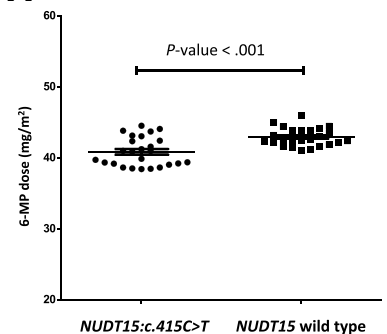

Figure 3 Associations between genetic variants and various blood counts as well as 6-MP administrative dosing among patients with acute leukemia undergoing 6-MP containing regimens. (A) ANC, (B) platelet count, (C) hemoglobin, and (D) daily 6-MP administrative dosing among patients with ITPA:c.94C>A polymorphism versus those with ITPA wide type. (E) ANC, (F) platelet count, (G) hemoglobin, and (H) daily 6-MP administrative dosing among patients with NUDTI5:c.4 I5C>T polymorphism versus those with NUDTI5 wide type.

Notes: Data in the graph are shown as dot plots. One dot plot represents a mean blood count or daily 6-MP administrative dosing on each cycle of treatment during the maintenance phase of leukemia treatment protocols. The two independent data sets were analyzed using independent sample $t$-test and Mann-Whitney $U$-test; $p$-value $<0.05$ was considered as statistically significant.

Abbreviations: ANC, absolute neutrophil count; ITPA, inosine triphosphate pyrophosphohydrolase; NUDTI5, nucleoside diphosphate linked moiety X-type motif I5; 6-MP, mercaptopurine.

monthly ANC during treatment $(p$-value $=0.061)$ (Table 4, Model 3). Overall frequency of myelotoxicity (neutropenia, anemia, and thrombocytopenia) from
1,635 episodes of CBC obtained on each monthly cycle of chemotherapy from participating patients was described in Table 5 .

Table 4 Effect of ITPA:c.94C>A and NUDTI5:c.4I5C>T Polymorphisms and 6-MP Dosing on ANC

\begin{tabular}{|c|c|c|c|c|}
\hline \multirow[t]{2}{*}{ ANC } & \multirow[t]{2}{*}{ Coefficient } & \multirow[t]{2}{*}{$p$-value } & \multicolumn{2}{|c|}{$95 \% \mathrm{Cl}$} \\
\hline & & & Lower & Upper \\
\hline \multicolumn{5}{|l|}{ Model I } \\
\hline ITPA:c.94C>A & 11.90 & 0.948 & -343.11 & 366.92 \\
\hline 6-MP dose & -8.40 & 0.062 & -17.22 & 0.42 \\
\hline \multicolumn{5}{|l|}{ Model 2} \\
\hline NUDTI5:C.4I5C>T & -463.81 & $0.004^{*}$ & -778.53 & -149.09 \\
\hline 6-MP dose & -9.29 & $0.038 *$ & -18.05 & -0.52 \\
\hline \multicolumn{5}{|l|}{ Model 3} \\
\hline ITPA:c.94C>A and NUDTI5:c.4I5C>T & -527.56 & $0.046^{*}$ & -1045.65 & -9.48 \\
\hline 6-MP dose & -8.41 & 0.061 & -17.19 & 0.38 \\
\hline
\end{tabular}

Notes: Longitudinal data from time-dependent variables were analyzed using multivariate GEE to examine the associations between ITPA and NUDTI5 polymorphisms as well as 6-MP administrative dosing and ANC measured monthly during the maintenance phase of acute leukemia protocols; ${ }^{*} p$-value $<0.05$ was considered as statistically significant.

Abbreviations: ANC, absolute neutrophil count; ITPA, inosine triphosphate pyrophosphohydrolase; NUDTI5, nucleoside diphosphate linked moiety X-type motif I5; 6-MP, mercaptopurine. 
Table 5 Overall Frequency of Myelotoxicity

\begin{tabular}{|c|c|c|c|c|c|c|}
\hline \multirow[t]{4}{*}{ CBC $(\mathrm{N}=1635)$} & \multicolumn{6}{|c|}{ Genetic Polymorphisms } \\
\hline & \multicolumn{2}{|c|}{ ITPA:c.94C>A } & \multicolumn{2}{|c|}{ NUDTI5:c.4I5C>T } & \multicolumn{2}{|c|}{ ITPA:c.94C>A and NUDTI5:c.4I5C>T } \\
\hline & Heterozygous & Wild type & Heterozygous & Wild type & Yes & No \\
\hline & $N=549$ & $N=1086$ & $\mathbf{N}=\mathbf{2 6}$ I & $N=1374$ & $\mathbf{N}=59$ & $N=1576$ \\
\hline \multicolumn{7}{|l|}{ ANC (cells $\left./ \mathbf{m m}^{3}\right)$} \\
\hline$<500$ & 23 & 65 & 25 & 63 & 3 & 85 \\
\hline $500-999$ & 90 & 146 & 47 & 189 & 14 & 222 \\
\hline$I, 000-1,500$ & 101 & 207 & 62 & 246 & 14 & 294 \\
\hline \multicolumn{7}{|c|}{ Hemoglobin (g/dL) } \\
\hline$<8.0$ & 4 & 21 & 5 & 11 & 1 & 15 \\
\hline $8.0-9.9$ & 60 & 111 & 34 & 137 & 4 & 167 \\
\hline $10-12$ & 285 & 484 & 149 & 620 & 38 & 731 \\
\hline \multicolumn{7}{|c|}{ Platelets (cells/mm ${ }^{3}$ ) } \\
\hline$<25,000$ & 3 & 3 & 0 & 6 & 0 & 6 \\
\hline $25,000-49,999$ & 2 & 8 & 3 & 7 & I & 9 \\
\hline $50,000-74,999$ & 3 & 8 & 0 & II & 0 & II \\
\hline $75,000-150,000$ & 42 & 100 & 19 & 123 & 7 & 135 \\
\hline
\end{tabular}

Abbreviations: ANC, absolute neutrophil count; CBC, complete blood count; ITPA, inosine triphosphate pyrophosphohydrolase; NUDTI5, nucleoside diphosphate linked moiety X-type motif 15.

\section{Discussion}

Pharmacogenetics has become one of the key elements in individualized cancer therapy over the past decades. ${ }^{22,23}$ Genetic variability of each individual patient could greatly affect drug metabolisms, therapeutic sensitivity, and risk for developing adverse effects. One of the common pharmacogenetic approaches to drug therapy is to identify single nucleotide polymorphisms or SNP and its impact on individual patient's phenotype as well as subsequent clinical consequences. ${ }^{24}$ The ultimate goal of this approach is to minimize treatment-related toxicity while preserving efficacy, while 6-MP is considered as the backbone of the maintenance phase of the acute lymphoblastic leukemia protocol. Several genetic polymorphisms of TPMT, ITPA, and NUDT15 have been identified and reported to be associated with drug metabolism, resulting in increasing drug-related myelotoxicity. ${ }^{25-28}$ Given geographic variation, TPMT polymorphisms were less reported in Asian populations. Conversely, NUDT15 and ITPA polymorphisms were found more in this specific population. ${ }^{13-18}$

Herein, we conducted a prospective cross-sectional observational study to evaluate the prevalence of NUDT15 and ITPA polymorphisms among Thai children with acute leukemia and further explore their association with 6-MP related myelotoxicity. Ninety-nine children receiving a diagnosis of acute leukemia as well as pediatric leukemia survivors were recruited and enrolled in this study. All patients participated and were involved to the end of the study with no loss of follow-up cases. The most common leukemia subtype diagnosis among participating patients in this study was pre-B ALL, which was in keeping with the common subtype prevalence among pediatric patients. Despite the diversity of acute leukemia subtypes, the recommended 6-MP administrative dosing on all treatment regimens was $50 \mathrm{mg} / \mathrm{m}^{2} /$ day. Interestingly, our PCR-RFLP method could identify ITPA:c.94C>A and NUDT15: c. $415 C>T$ polymorphisms among up to one half of the patients although all carried heterozygous genotypes. In addition, the prevalence of ITPA:c. $94 C>A$ and NUDT15: c. $415 C>T$ polymorphisms among patients in this study was 34 and $17 \%$, respectively, in which $4 \%$ of the patients were found to have co-occurrence of these two genetic polymorphisms. These results affirm the commonness of ITPA and NUDT15 polymorphisms in Asian compared with Caucasian populations ${ }^{16-18,29,30}$ although a higher incidence of ITPA polymorphism was observed in our study compared with $5-7 \%$ in Caucasian and up to $15 \%$ in Asian populations. ${ }^{31}$ We then further investigated potential associated factors including patient demographic data, disease information and treatment response among patients with and without ITPA:c.94C $>A$ and NUDT15:c.415C>T 
polymorphisms and found no differences in the demographic information between patients with and without polymorphisms. However, ITPA:c.94C $>A$ and NUDT15:c.415C>T polymorphisms, involved in 6-MP metabolisms, potentially increase the risk of developing drug-related myelotoxicity resulting in delayed cycles of chemotherapy. Additionally, they possibly increase the risk of disease relapse. The insignificant difference of treatment response between the two groups of patients in our study could be from careful monitoring of $\mathrm{CBC}$ measured monthly during the maintenance phase of treatment and adequate adjustment of 6-MP dosing based on ANC instead of holding off on treatment.

Associations between ITPA and NUDT15 genotypes and 6-MP related myelotoxicity were further explored. Interestingly, suppression of granulopoiesis and megakaryopoiesis was remarkable among patients presenting the NUDT15:c.415C $>T$ polymorphism, but not among those carrying the ITPA:c.94C $>A$ polymorphism. This result was confirmed by significantly decreased 6-MP administrative dosing among patients presenting the NUDT15:c.415C $>T$ polymorphism. However, the significant decrease of 6-MP administrative dosing among patients carrying the ITPA wild type could be from patients presenting NUDT15: c. $415 C>T$ being included in this group. Multivariate GEE was used to evaluate the effect of ITPA:c.94C $>A$ and NUDT15:c.415C>T polymorphisms as well as 6-MP administrative dosing among patients carrying these specific polymorphisms on monthly ANC longitudinally measured during the maintenance phase of acute leukemia treatment protocols. The result of our study affirmed the significant negative relationship between NUDT15: c. $415 C>T$ polymorphism and neutropenia ( $p$ value $=0.004$ ) in which patients carrying this specific polymorphism would have ANC 463 cells $/ \mathrm{mm}^{3}$ lower than those carrying the NUDT15 wild type. In addition, ANC among patients carrying the NUDT15:c.415C $>T$ polymorphism would decrease by a decrement of 9 cells $/ \mathrm{mm}^{3}$ on every $1 \mathrm{mg}$ increased 6-MP dose ( $p$-value=0.038). Interestingly, the effect of ITPA:c.94C $>A$ polymorphism and 6-MP administrative dosing among patients carrying this specific polymorphism were not significantly evident in our study. These findings were consistent with recent studies among Japanese and Chinese children in which no differences were found between 6-MP dose administrative dosing and ITPA polymorphisms. ${ }^{16,32}$ Moreover, we found a significant negative relationship between co-occurrence of ITPA:c.94C $>A$ and NUDT15:c.415C $>T$ polymorphisms and neutropenia ( $p$-value $=0.046)$ in which patients having co-occurrence of these two polymorphisms would have ANC 527 cells $/ \mathrm{mm}^{3}$ lower than that of wild type patients. However, the effect of 6-MP administrative dosing was insignificant which might have stemmed from this dilutional effect of the ITPA polymorphism group. According to Table 5, there were certain numbers of patients who did not carry ITPA:c.94C $>A$ and NUDT15:c.415C $>T$ polymorphisms and developed myelotoxicity. The development of myelotoxicity among those patients could be from several factors such as TPMT polymorphisms which were not identified in this study, a direct myelosuppressive effect from 6-MP itself or other chemotherapy coadministered with 6-MP in the treatment regimen, less bone marrow reserve from previous treatment prior to entering the maintenance phase or recent infection-associated bone marrow suppression.

The limitations of this study included the small sample size of participating patients, which might have contributed to insignificant differences of some results. In addition, data obtained from the unique and specific populations in this study might not be generally applicable to all patients at different age ranges and ethnic groups. Moreover, the correlation between genetic polymorphisms and treatment interruption as well as total duration of neutropenia not examined in our study needs to be further explored. Since TPMT polymorphisms were less reported in Asian populations, we did not include evaluation of TPMT polymorphisms in our study.

\section{Conclusion}

Although the incidence of ITPA:c.94C $>A$ polymorphism among Thai children with acute leukemia in our study was higher than that reported from other Asian countries, ITPA genotyping could not be used as a predictor for 6-MP induced myelotoxicity. In contrast, the incidence NUDT15:c. $415 C>T$ polymorphism in our study was comparable to other Asian countries and a strong association was also observed between either NUDT15 polymorphism alone or combined with ITPA polymorphism and developing 6-MP induced myelotoxicity.

\section{Abbreviations}

ALL, acute lymphoblastic leukemia; ANC, absolute neutrophil count; APL, acute promyelocytic leukemia; $\mathrm{CBC}$, complete blood count; CI, confidence interval; CNS, central nervous system; GEE, generalized estimating equation; ITPA, inosine triphosphate pyrophosphohydrolase; MPAL, mixed phenotype acute leukemia; NUDT15, nucleoside diphosphate linked moiety X-type motif 15 ; PCR-RFLP, PCR-Restriction Fragment Length 
Polymorphism; PROBE, Preferred Reporting items for OBservational studies in Endodontics; SD, standard deviation; ThaiPOG, Thai Pediatric Oncology Group; TPMT, thiopurine methyltransferase; 6-MP, 6-mercaptopurine.

\section{Data Sharing Statement}

The data that support the findings of this study are available on reasonable request from the corresponding author. The data are not publicly available due to privacy or ethical restrictions.

\section{Acknowledgments}

The funding from the Phramongkutklao College of Medicine, Royal Thai Army was used to conduct the study, analyze, and interpret the study results and submit the study for publication. The authors would like to thank the patients and families for participating in the study.

\section{Author Contributions}

All authors made substantial contributions to the conception and design, acquisition of data, or analysis and interpretation of data; took part in drafting the article or revising it critically for important intellectual content; agreed to submit to the current journal; gave final approval of the version to be published; and agree to be accountable for all aspects of the work.

\section{Disclosure}

The authors report no conflicts of interest in this work.

\section{References}

1. Allemani C, Matsuda T, Di Carlo V, et al. Global surveillance of trends in cancer survival 2000-14 (CONCORD-3): analysis of individual records for 37513025 patients diagnosed with one of 18 cancers from 322 population-based registries in 71 countries. Lancet. 2018;391 (10125):1023-1075.

2. Song TY, Lee SH, Kim G, Baek HJ, Hwang TJ, Kook H. Improvement of treatment outcome over 2 decades in children with acute myeloid leukemia. Blood Res. 2018;53(1):25-34. doi:10.5045/br.2018.53.1.25

3. Shamoon RP, Ali MD, Shabila NP. Overview and outcome of Hodgkin's lymphoma: experience of a single developing country's oncology centre. PLoS One. 2018;13(4):e0195629. doi:10.1371/journal.pone. 0195629

4. Inaba H, Mullighan CG. Pediatric acute lymphoblastic leukemia. Haematologica. 2020;105(11):2524-2539. doi:10.3324/ haematol.2020.247031

5. Fu Y, Wang H, Zhai X, et al. Improved outcome of newly diagnosed childhood mature B-cell lymphoma/leukemia with high tumor burden treated with BFM95-based protocol combining rituximab: a report from Shanghai, China. J Pediatr Hematol Oncol. 2019;41(3):170174. doi:10.1097/MPH.0000000000001419

6. Vento S, Cainelli F. Infections in patients with cancer undergoing chemotherapy: aetiology, prevention, and treatment. Lancet Oncol. 2003;4(10):595-604. doi:10.1016/S1470-2045(03)01218-X
7. Rusu RA, Sirbu D, Curseu D, et al. Chemotherapy-related infectious complications in patients with Hematologic malignancies. J Res Med Sci. 2018;23:68. doi:10.4103/jrms.JRMS_960_17

8. Nelson JA, Carpenter JW, Rose LM, Adamson DJ. Mechanisms of action of 6-thioguanine, 6-mercaptopurine, and 8-azaguanine. Cancer Res. 1975;35(10):2872-2878.

9. Bostrom B, Erdmann G. Cellular pharmacology of 6-mercaptopurine in acute lymphoblastic leukemia. Am J Pediatr Hematol Oncol. 1993;15(1):80-86. doi:10.1097/00043426-199302000-00010

10. Ma XL, Wang B, Guo HY, et al. [Tolerability of 6-mercaptopurine in children with acute lymphoblastic leukemia]. Zhonghua Er Ke Za Zhi. 2010;48(4):289-292. [Chinese].

11. Albayrak M, Konyssova U, Kaya Z, et al. Thiopurine methyltransferase polymorphisms and mercaptopurine tolerance in Turkish children with acute lymphoblastic leukemia. Cancer Chemother Pharmacol. 2011;68(5):1155-1159. doi:10.1007/s00280-011-1599-7

12. Adam de Beaumais T, Jacqz-Aigrain E. Pharmacogenetic determinants of mercaptopurine disposition in children with acute lymphoblastic leukemia. Eur J Clin Pharmacol. 2012;68(9):1233-1242. doi:10.1007/s00228-012-1251-4

13. Hiratsuka M, Inoue T, Omori F, Agatsuma Y, Mizugaki M. Genetic analysis of thiopurine methyltransferase polymorphism in a Japanese population. Mutat Res. 2000;448(1):91-95. doi:10.1016/S0027-5107 (00)00004-X

14. Collie-Duguid ES, Pritchard SC, Powrie RH, et al. The frequency and distribution of thiopurine methyltransferase alleles in Caucasian and Asian populations. Pharmacogenetics. 1999;9(1):37-42. doi:10.1097/ 00008571-199902000-00006

15. Chang JG, Lee LS, Chen CM, et al. Molecular analysis of thiopurine S-methyltransferase alleles in South-east Asian populations. Pharmacogenetics. 2002;12(3):191-195. doi:10.1097/00008571200204000-00003

16. Zhou H, Li L, Yang P, et al. Optimal predictor for 6-mercaptopurine intolerance in Chinese children with acute lymphoblastic leukemia: NUDT15, TPMT, or ITPA genetic variants? BMC Cancer. 2018;18 (1):516. doi:10.1186/s12885-018-4398-2

17. Maeda T, Sumi S, Ueta A, et al. Genetic basis of inosine triphosphate pyrophosphohydrolase deficiency in the Japanese population. Mol Genet Metab. 2005;85(4):271-279. doi:10.1016/j.ymgme.2005.03.011

18. Khera S, Trehan A, Bhatia P, Singh M, Bansal D, Varma N. Prevalence of TPMT, ITPA and NUDT 15 genetic polymorphisms and their relation to $6 \mathrm{MP}$ toxicity in north Indian children with acute lymphoblastic leukemia. Cancer Chemother Pharmacol. 2019;83 (2):341-348. doi:10.1007/s00280-018-3732-3

19. Gerbek T, Ebbesen M, Nersting J, Frandsen TL, Appell ML, Schmiegelow K. Role of TPMT and ITPA variants in mercaptopurine disposition. Cancer Chemother Pharmacol. 2018;81(3):579-586. doi:10.1007/s00280-018-3525-8

20. Cao H, Hegele RA. DNA polymorphisms in ITPA including basis of inosine triphosphatase deficiency. J Hum Genet. 2002;47(11):620622. doi: $10.1007 / \mathrm{s} 100380200095$

21. Moriyama T, Nishii R, Perez-Andreu V, et al. NUDT15 polymorphisms alter thiopurine metabolism and hematopoietic toxicity. Nat Genet. 2016;48(4):367-373. doi:10.1038/ng.3508

22. Lu DY, Lu TR, Xu B, Ding J. Pharmacogenetics of cancer therapy: breakthroughs from beyond? Future Sci OA. 2015;1(4):FSO80. doi: $10.4155 /$ fso. 15.80

23. Relling MV, Dervieux T. Pharmacogenetics and cancer therapy. Nat Rev Cancer. 2001;1(2):99-108. doi:10.1038/35101056

24. Erichsen HC, Chanock SJ. SNPs in cancer research and treatment. $\mathrm{Br}$ J Cancer. 2004;90(4):747-751. doi:10.1038/sj.bjc.6601574

25. Moradveisi B, Muwakkit S, Zamani F, Ghaderi E, Mohammadi E, Zgheib NK. ITPA, TPMT, and NUDT15 genetic polymorphisms predict 6-mercaptopurine toxicity in middle eastern children with acute lymphoblastic leukemia. Front Pharmacol. 2019;10:916. doi:10.3389/fphar.2019.00916 
26. Singh M, Bhatia P, Khera S, Trehan A. Emerging role of NUDT15 polymorphisms in 6-mercaptopurine metabolism and dose related toxicity in acute lymphoblastic leukaemia. Leuk Res. 2017;62:1722. doi:10.1016/j.leukres.2017.09.012

27. Stocco G, Crews KR, Evans WE. Genetic polymorphism of inosine-triphosphate-pyrophosphatase influences mercaptopurine metabolism and toxicity during treatment of acute lymphoblastic leukemia individualized for thiopurine-S-methyl-transferase status. Expert Opin Drug Saf. 2010;9(1):23-37. doi:10.1517/14740330 903426151

28. Wahlund M, Nilsson A, Kahlin AZ, et al. The role of TPMT, ITPA, and NUDT15 variants during mercaptopurine treatment of Swedish pediatric patients with acute lymphoblastic leukemia. $J$ Pediatr. $\quad 2020 ; 216: 150-7 \quad$ e1. doi:10.1016/j.jpeds.2019.09. 024
29. Liang DC, Yang CP, Liu HC, et al. NUDT15 gene polymorphism related to mercaptopurine intolerance in Taiwan Chinese children with acute lymphoblastic leukemia. Pharmacogenomics $J$. 2016;16 (6):536-539. doi:10.1038/tpj.2015.75

30. Zhu X, Wang XD, Chao K, et al. NUDT15 polymorphisms are better than thiopurine S-methyltransferase as predictor of risk for thiopurineinduced leukopenia in Chinese patients with Crohn's disease. Aliment Pharmacol Ther. 2016;44(9):967-975. doi:10.1111/apt.13796

31. Roberts RL, Barclay ML. Update on thiopurine pharmacogenetics in inflammatory bowel disease. Pharmacogenomics. 2015;16(8):891903. doi:10.2217/pgs.15.29

32. Tanaka Y, Kato M, Hasegawa D, et al. Susceptibility to 6-MP toxicity conferred by a NUDT15 variant in Japanese children with acute lymphoblastic leukaemia. Br J Haematol. 2015;171(1):109-115. doi:10.1111/ bjh. 13518

\section{Publish your work in this journal}

The Application of Clinical Genetics is an international, peerreviewed open access journal that welcomes laboratory and clinical findings in the field of human genetics. Specific topics include: Population genetics; Functional genetics; Natural history of genetic disease; Management of genetic disease; Mechanisms of genetic disease;
Counselling and ethical issues; Animal models; Pharmacogenetics; Prenatal diagnosis; Dysmorphology. The manuscript management system is completely online and includes a very quick and fair peerreview system, which is all easy to use. Visit http://www.dovepress. com/testimonials.php to read real quotes from published authors. 